\title{
Vergleich solarer Erscheinungen mit der Veränderlichkeit von Sternen in Gasnebeln
}

\author{
Von Hans-Jost BingE* \\ (Z. Naturforschg. $\mathbf{7}$ a, 440-444 [1952]; eingegangen am 24. Januar 1952)
}

\begin{abstract}
Die vorliegenden Untersuchungen über die RW Aurigae-Veränderlichen machen es wahrscheinlich, daß diese Sterne einen sonnenähnlichen Charakter haben. Dadurch liegt es nahe, die Besonderheiten dieser Sterne durch eine der Fleckenaktivität der Sonne analoge, aber viel stärkere Erscheinung zu erklären. Es wird in dieser Arbeit gezeigt, daß die Beobachtungen sich dadurch gut verstehen lassen.

Im Anschluß daran wird versucht, die Aktivität der Sonne durch äußere Einflüsse, wie sie für die RW Aurigae-Sterne angenommen werden, auf mechanischem Wege zu erklären.
\end{abstract}

$\mathrm{E}$ $\mathrm{s}$ ist eine alte Frage, ob die Sonnenflecken eine Art schwache Veränderlichkeit der Sonne hervorrufen. Vor etwa 80 Jahren nahm Zöllner an, daß die Sonnenflecken Schlackenbildungen, also Vorboten einer allmählichen Abkühlung der Sonne wären. Er behauptete ferner, daß bei weiterer Abkühlung diese Flecken immer ausgedehnter würden und schließlich zu einem Mira-Lichtwechsel führen könnten. Bei der weiteren Entwicklung der Astrophysik wurde diese primitive Anschauung aufgegeben, denn es stellte sich heraus, daß die MiraSterne und andere rote Veränderliche ( $\mu$ Cephei) Riesen sind. Außerdem wurde durch die Hypothesen von Faye, v. Oppolzer und Bjerknes, welche durch die spektro-heliographischen Aufnahmen von Hale und anderen gestützt wurden, dem Abkühlungscharakter des Fleckenphänomens jeder Boden entzogen

Wenn man heute das Fleckenphänomen im Sinne einer Veränderlichkeit der Sonne untersuchen will, muß man sich vor allem die Stellung der Sonne im Russell-Diagramm vor Augen halten und sich fragen, welcheVeränderlichkeit überhaupt bei einem Hauptreihenstern des Spektraltyps GO zu erwarten sein kann. Danach scheiden sofort Mira-, $\delta$ Cephei-, RV Tauri- und RR Lyrae-Veränderlichkeit aus, da es sich bei allen diesen um Riesen handelt; ebenso $\mu$ Cephei und $\mathrm{R}$ Coronae borealis.

Es bleibt also nur die sog. Zwergveränderlichkeit übrig. Unter den veränderlichen Sternen dieser Art scheiden natürlich sofort Sterne wie die Novae, die Nova-ähnlichen, die U Geminorum- und Z Camelopardalis-Sterne aus, da
1. der Lichtwechsel dieser Sterne in Formen erfolgt, die von denen bei der Sonne bekannten Erscheinungen gänzlich verschieden sind und

2. es sich wahrscheinlich um Übergänge von der Hauptreihe zu den Weißen Zwergen handelt.

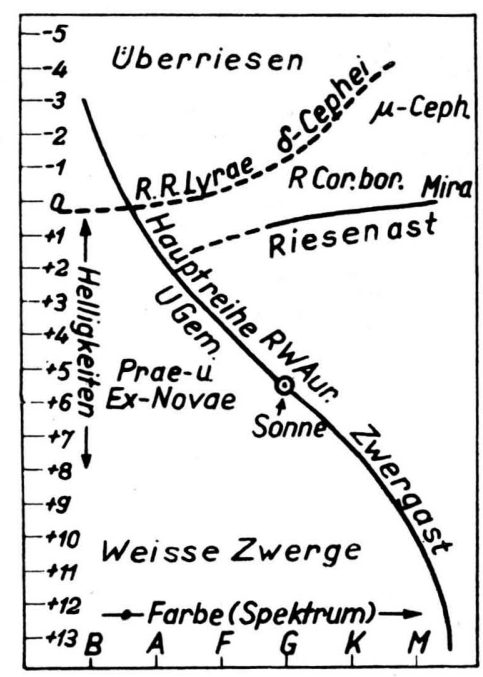

Abb. 1. Lage derVeränderlichen im Russell-Diagramm (nach H. U. Sandig).

Bei echten Hauptreihensternen kommen wohl nur die folgenden Arten von sog. Nebelveränderlichen ${ }^{1}$ vor:

* Hamburg 24, Graumannsweg 50.

1 Die Einteilung stammt von K. Himpel, Himmelswelt 52, 94 [1942]. 
I. B-Sterne und A-Sterne bis etwa A 3 werden durch Nebel zu zeitlich sehr ausgedehnten Minima (von der Größenordnung 10 Jahre) angeregt, wobei das Spektrum häufig Emissionslinien zeigt. (Z. B.: X Persei, RX Puppis.)

II.Von etwa A 5 an bis GO sind die Minima häufig nur noch von der Dauer weniger Tage bis Wochen, aber es treten keine Aufhellungen über das normale Maximallicht auf. Über die Spektra ist wenig bekannt. (Z. B.: T Orionis, BN Orionis, RY Orionis und RY Tauri.)

III. dG- bis dM-Sterne werden durch den Nebel völlig gestört, so daß Z Camelopardalis-ähnliche Lichtausbrüche mit BN Orionis-artigen Minima in unregelmäßiger Folge abwechseln. Das Spektrum zeigt häufig helle Linien. Diese Sterne

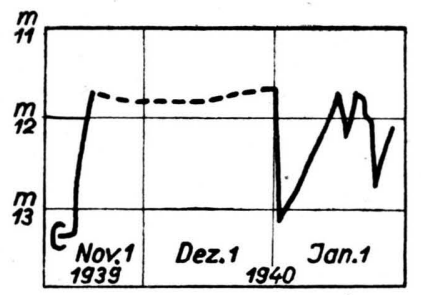

Abb. 2a. Teil der Lichtkurve von RW Aurigae nach Himpel ${ }^{1}$.

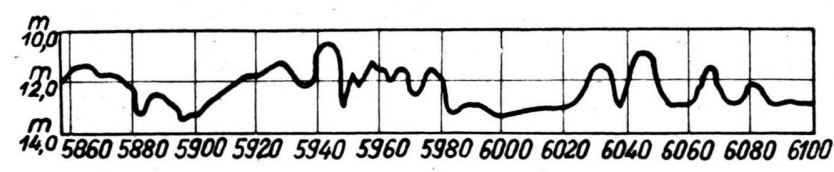

Abb. 2 b. Lichtkurve von RR Tauri nach L. J a cchia*. Zu Abb. 2 a und 2 b. RW Aurigae und RR Tauri haben beide annähernd den gleichen Spektraltyp wie die Sonne $(\mathrm{dGO})$ und zeigen den typischen raschen Lichtwechsel. sind die unruhigsten und unregelmäßigsten von allen Veränderlichen. (Z. B.: RW Aurigae, T Tauri, RU Lupi, R Coronae australis, Y Leporis und UZ Tauri ${ }^{2}$.

Besteht nun eine Ähnlichkeit zwischen den beiden letzteren Gruppen von Hauptreihen-Veränderlichkeit und dem Sonnenfleckenphänomen? Zunächst ist dazu zu sagen, daß die täglichen SonnenfleckenRelativżahlen häufig äußerst raschen und unregelmäßigen Schwankungen unterliegen, wie wir sie auch aus den Lichtkurven der RW Aurigae-Sterne kennen. Da nun Flecke weniger Licht emittieren als der ungestörte Teil der Sonnenoberfläche, so ist die Kurve der Relativzahlen gewissermaßen die umgekehrte Lichtkurve der Sonne. Spiegelt man die Sonnenfleckenkurve an der Zeitachse, so erhält man ein ungefähres Bild der Lichtkurve. Wie dieses Bild zeigt, würde bei entsprechender Verstärkung der Veränderlichkeit die Sonne als RW AurigaeStern klassifiziert werden müssen.

Da im Sonnenflecken-Minimum die Kurve der Relativzahlen einen ruhigeren Verlauf hat, ist der Ruhezustand der Veränderlichen mit dem Fleckenminimum gleichzusetzen. Bei T Orionis, BN Orionis und einigen ähnlichen Sternen fällt nun der Ruhezustand mit dem hellen Licht zusammen. Daraus muß gefolgert werden, daß das helle Licht dem Fleckenminimum entspricht.

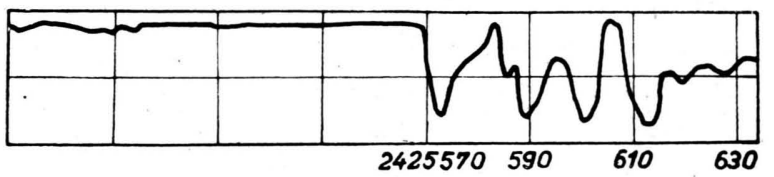

Abb. 3a. Plötzliches Einsetzen derVeränderlichkeit bei T Orionis (nach L. Jacchia)*.

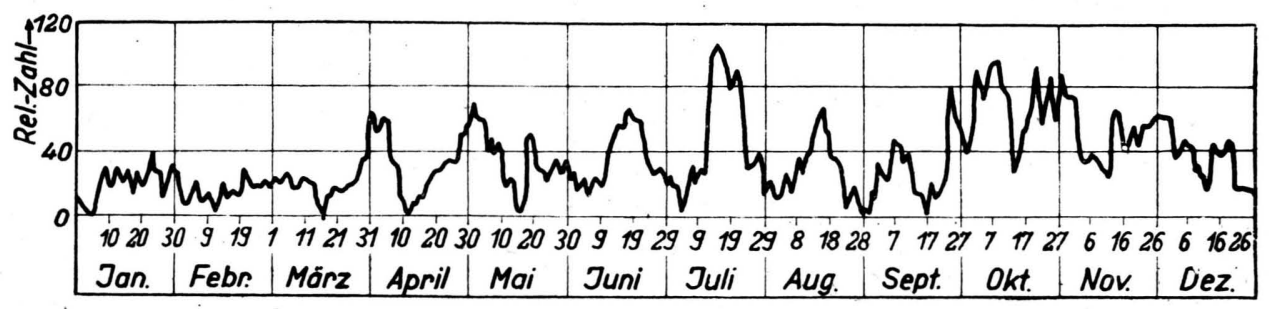

Abb. 3b. Tägliche Flecken-Relativzahlen für das Jahr 1945 nach der Züricher Statistik. (Astronom. Mitt. Zürich Nr. 148.) Man beachte besonders die plötzliche Verstärkung der Fleckentätigkeit Ende März.

2 Bzgl. der Spektra vgl. H. Ludendorff, Handb. Astrophysik Bd. VI. Ferner A. Joy, Astrophysic. J. 102. $168[1945]$.
* Die Abbildungen $2 \mathrm{~b}$ und $3 \mathrm{a}$ sind der Arbeit ,La stella variabili" in den Veröffentlichungen des Astronom. Obs. Univ. Bologna Bd. II Nr. 1-16 [1929 bis 1934] entnommen). 
Nehmen wir also einmal an, daß die RW AurigaeSterne G- bis M-Sterne der Hauptreihe seien, die durch Nebel zur Fleckenbildung angeregt werden, so erheben sich vier schwerwiegende Fragen:

1. Sind die spektralen Anomalien mancher RW Aurigae-Sterne durch diese Hypothese erklärbar ?

2. Gibt es RW Aurigae-Sterne mit so kleiner Amplitude, daß sie die Analogie zum gegenwärtigen Zustand der Sonne wahrscheinlich machen?

3. Kann für die Fleckenbildung auf der Sonne ein sie umgebender Nebel verantwortlich gemacht werden?

4. Wenn ja, wie ist dann der Mechanismus der Fleckenbildung zu deuten?

Bzgl. Frage 1 muß gesagt werden, daß in Verbindung mit den Flecken auf der Sonne häufig Energieausbrüche vorkommen, welche z. T. mit Materialausbrüchen verbunden - ein Emissionsspektrum ${ }^{3}$ zeigen, das in seiner qualitativen Zusammensetzung den Emissionslinien der Spektra von T Tauri, RW Aurigae, RU Lupi und $\mathrm{R}$ Coronae australis entspricht (hauptsächlich Linien von $\mathrm{H}, \mathrm{He}$, Ca I, Ca II, Fe I, Fe II, Ti II, Cr I, Cr II und einigen anderen Elementen, z. B. Na I und Sc II). Bei den Sternen mit permanenten Emissionsspektra braucht man nur anzunehmen, daß dauernd heftige Eruptionen erfolgen.

$\mathrm{Zu}$ Frage 2 ist zu bemerken, daß Hoffmeister bei seinen Felderplanarbeiten eine ganze Reihe von RW Aurigae-Sternen sehr kleiner Amplitude gefunden hat. Auch diese Sterne zeigen vorzugsweise G-Spektra. Ihre Spektra streuen allerdings zwischen etwa AO und M. Da aber die roten RW AurigaeSterne wie Y Leporis auch zur Hauptreihe gehören ${ }^{4}$, kann angenommen werden, daß alle RW Aurigae-ähnlichen Typen Hauptreihensterne von sonnenähnlicher Konstitution sind.

Zur Frage 3 ist zu sagen, daß schon allein das Zodiakallicht beweist, daß unsere Sonne von einer

\footnotetext{
${ }^{3}$ Vgl. R. S. Richardson u. R. Minkowski, Astrophysic. J. 89, 847 [1939]; C. W. Allen, Monthly Notices Roy. astronom. Soc. 100, 635 [1940]; M. A. Ellison, Monthly Notices Roy. astronom. Soc. 106, 500 [1946].

${ }^{4}$ Auf Grund theoretischer Überlegungen erscheint es dem Verf. absolut unwahrscheinlich, daß M-Riesen im Nebel rasche Veränderlichkeit großer Amplitude zeigen. Vielmehr ist bei roten Riesen im Nebel eine langsame Veränderlichkeit kleiner Amplitude zu erwarten, wie K. Himpel das ja auch bei T Carinae und W Serpentis nachgewiesen hat (vgl. Himmelswelt 52, 94 [1942]).
}

Wolke interstellarer Materie umgeben ist. Biermann vertritt den Standpunkt ${ }^{5}$, daß diese Materie sogar dichter ist, als man früher annahm. Es dürfte denkbar sein, daß die Sonne immer noch in einem dünnen Ausläufer des Nölkeschen Eiszeitnebels ${ }^{6}$ steht. Daher wäre auch von dieser Seite derVergleich mit den Nebelveränderlichen zulässig.

Mit Frage 4 schneidet man ein sehr heikles Problem an, das allerdings nunmehr unter einem gänzlich neuen Gesichtswinkel erscheint. Es sei hier gestattet, eine Ansicht vorzutragen, die der oben wahrscheinlich gemachten Identität zwischen der Fleckenbildung und einer Art Nebelveränderlichkeit (RW Aurigae- oder BN Orionis-Veränderlichkeit) Rechnung trägt. Die Ursache der Fleckenbildung wird bei dieser Annahme auf die Wechselwirkung zwischen Stern und Nebel zurückgeführt. Wie kann man sich diese Wechselwirkung veranschaulichen?

Wie schon von verschiedenen Autoren ${ }^{7}$ ausgeführt worden ist, bildet sich um einen Stern im Nebel eine sehr ausgedehnte Verdichtung von Nebelmaterie. In dieser Hülle liegt nun der Mechanismus der Veränderlichkeit. Denn, da der Stern nicht im Nebel ruht, sondern einerseits eine fortschreitende Bewegung ausführt, andererseits aber um seine Achse rotiert und - falls Planeten vorhanden - auch von diesen beeinflußt wird, treten in der Nebelhülle Strömungen und Turbulenzen auf, welche sehr heftig sein können. Nölke hat u. a. bei seiner Erklärung des Kometeneinfangs die bei einer reinen Translationsbewegung auftretenden Erscheinungen untersucht. Er kommt zu dem Resultat, daß sich hinter der Sonne ein Schweif aus verdichteter Nebelmaterie bildet und die Sonne selbst von einer Nebelhülle umgeben wird. In diesem Schweif und in der Hülle wirbelt die verdichtete Nebelmaterie chaotisch durcheinander. Die Geschwindigkeit dieser Turbulenz unmittelbar an der Sternoberfläche ist nach Nölke von der Größenordnung

5 Nach briefl. Mitt. an den Verfasser.

${ }^{6}$ Vgl. K. Himpel, Z. Naturforschg. 2a, 425 [1947]; C. Hoffmeister, S.-B. preuß. Akad. Wiss., physik.math. Kl. 18 [1936]; W. Becker, Z. Astrophysik 11, 89 [1935], Fortschr. Astronom. 1, [1938].

${ }^{7}$ Vgl. K.Himpel ${ }^{1}$; M.C.J ohnson, Monthly Notices Roy. Astronom. Soc. 88, 392 [1928]; H. Ludendorff, Handb. Astrophysik Bd. VI, S. 82; E.W. Brown, Astrophysic. J. 53, 169 [1921]; F. Nölke, Das Problem der Entstehung unseres Planetensystems $\S 158$ [1919]. 
der parabolischen Geschwindigkeit, die bei der Sonne etwa $620 \mathrm{~km} / \mathrm{sec}$ beträgt. Im Gebiet der inneren Korona werden nun tatsächlich auch Strömungen von der Größenordnung einiger $100 \mathrm{~km} / \mathrm{sec}$ beobachtet und zwar an den Protuberanzen. Wenn auch für die eruptiven Protuberanzen der Strahlungsdruck (namentlich des Lyman-Kontinuums - nach Unsöld) verantwortlich gemacht werden kann, so treten doch Bewegungen auf, die nur durch Turbulenz erklärbar sind. Als Beispiele seien nur die Tornado-Protuberanzen und diejenigen Protuberanzen angeführt, welche in einen Fleck hineingesogen werden. Wie Filmaufnahmen beweisen, sind diese Vorgänge weit verbreitet und stellen ein Charakteristikum der Protuberanzen überhaupt dar. Außerdem werden die Protuberanzen manchmal von Gebieten mit völlig ungestörter Photosphäre angesogen. Gerade die letzte Erscheinung erweckt den Eindruck, daß in der inneren Korona Kräfte wirksam sind, die nicht im Sonnenkörper selbst ihren Ursprung haben.

Man könnte nach alledem erwarten, daß sich bei stärkerer Nebelveränderlichkeit auch die Protuberanzen stärker bemerkbar machen. Daß dies wirklich der Fall ist, scheint der Stern AB Aurigae zu beweisen. Dieser Stern steht nach Shapley ${ }^{8}$ in einem Dunkelgebiet und gehört zur ersten der oben angeführten Klassen von Nebenveränderlichen (Spektrum AOep). Über die breiten dunklen Wasserstofflinien des AO-Spektrums lagern sich schmale helle Komponenten, welche gegen die Absorptionslinien um wechselnde Beträge von der
Größenordnung $100 \mathrm{~km} / \mathrm{sec}$ verschoben erscheinen. Ein Doppelstern scheint nicht vorzuliegen, da andere Linien normal sind, und Merrill ${ }^{9}$ kommt zu dem Schluß, daß vermutlich intensive Protuberanzen als Quelle der verschobenen H-Emissionslinien anzunehmen sind. Er schreibt wörtlich: „Indeed, a curious parallelism between typical features of $\mathrm{Be}$ Spectra and certain phenomena of the solar chromosphere indicates a degree of similarity between the physical conditions in atmospheres of Be-stars and those in the chromosphere and prominences and suggests, by way of speculations, that the (unknown) cause of rapid motion in prominences may perhaps, in stars hotter then the sun, be sufficiently powerful and well organised to produce observable effects in the spectrum of the integrated light".

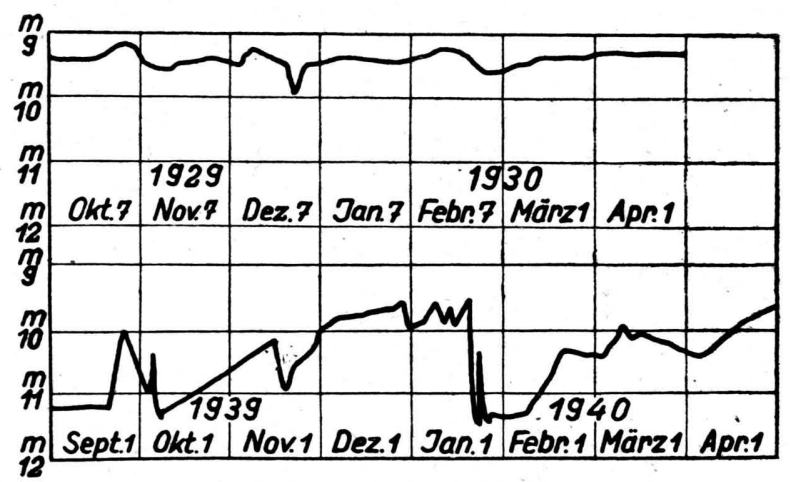

Abb. 4. Lichtkurve von BN Orionis (Spektrum A 7), oben Ruhezustand, unten Zustand starker Veränderlichkeit; nach Himpel ${ }^{1}$.

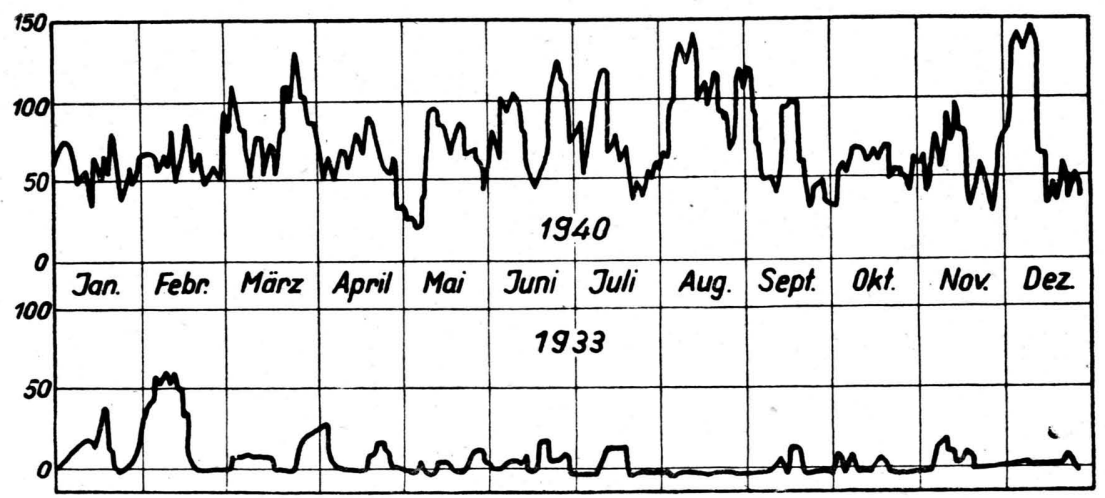

Abb. 5. Ruhiger (1933) und unruhiger (1940) Verlauf der Sonnenflecken-Relativzahlen (nach den Astronom. Mitt. Zürich).

${ }^{8}$ Vgl. H. Shapley, Harvard Bull. 1924, 798; Handb. Astrophysik Bd.VI Kap. 2 Ziff. 13.
${ }^{9}$ Vgl. P. W. Merrill, Astrophysic. J. 77, 103 [1933]. 
Wenn diese Ansicht über die Be-Sterne richtig ist, so bedeutet die Tatsache, daß eine Anzahl solcher Sterne mit komplexen Balmer-Linien $(\gamma$ Cassiopeiae, Pleione, $\mathrm{R}$ Monocerotis, $\mathrm{S}$ Doradus, AG Carinae) Beziehungen zu Nebeln haben, eine Stütze unserer Auffassung über die Natur der Bewegungen in den Protuberanzen.

Da nun die Protuberanzen in gewissem Maße mit der Fleckenbildung zusammenhängen, spricht diese Überlegung für die vorgetragene Hypothese ïber die Natur der Nebelveränderlichkeit.

Über die Entstehung einer bipolaren Fleckengruppe kann man sich nun folgende Vorstellung machen.

Ein Wirbelfaden aus der Korona wandert in den Sonnenkörper ein. Seine Eintritt- und Austrittstelle bilden die Flecke einer bipolaren Gruppe. Da nun nach v. Oppolzer ${ }^{10}$ der eindringende Wirbel sich erhitzt, so nimmt die Opazität der Photosphäre im Wirbelfaden zu, wodurch in diesem Gebiet weniger Energie nach außen gelangt (vgl. Johnson ${ }^{7}$ ). Dadurch vermindert sich die Oberflächentemperatur, so daß das G-Spektrum der Sonne im Kernfleck in ein K-Spektrum übergeht und dieses Gebiet als dunkler Fleck auf der Sonnenscheibe erscheint.

Damit stimmt überein, daß kurz vor der Bildung einer Fleckengruppe die Chromosphäre an der Stelle des künftigen Flecks besonders hell aufleuchtet. Dies ist ein Zeichen dafür, daß einmal die Fleckenbildung von außen nach innen fortschreitet und sie zweitens mit einer anfänglichen Erhitzung ${ }^{11}$ beginnt.

\footnotetext{
10 Selbst wenn man der Meinung ist, daß die thermodynamischen Voraussetzungen der Oppolzerschen Theorie nicht zutreffen, so kann man dennoch leicht eine Überhitzung des Fleckenwirbels dadurch erhalten, daß die elektrisch leitende Gasmasse in einem Magnetfeld rotiert und sich dabei Wirbelströme bilden, welche den Wirbel aufheizen.

${ }^{11}$ In diesem Zusammenhang dürften auch die Beziehungen zwischen den $\mathbf{R}$ Coronae borealis-Sternen und den Nebelveränderlichen deutbar sein. Bei beiden Gruppen wird durch Erhitzung der Photosphäre die Opazität gesteigert, was eine Abnahme der Ausstrahlung zur Folge hat. Der einzige Unterschied besteht
}

Leider bietet diese Theorie noch keine Erklärung für die elfjährige Periode und für den Wechsel in der Polarität der Sonnenflecke. Es sind aber einige Anhaltspunkte dafür vorhanden, daß auch bei $\mathrm{T}$ Orionis, BN Orionis, T Tauri, X Persei usw. Zeiten der Unruhe mit solchen der Ruhe wechseln. Es muß das Ziel weiterer Untersuchungen sein, bei den Nebelveränderlichen Erscheinungen festzustellen, die der elfjährigen Periode entsprechen. Als Ursache für diese Erscheinungeñ dürften wohl auch rein mechanische Vorgänge in der Nebelhülle - etwa die Gravitationswirkung von Planeten - in Frage kommen.

Da bei den chromosphärischen Eruptionen außer der sichtbaren Strahlung und der UV-Strahlung im Lyman-Gebiet auch eine starke RadiofrequenzStrahlung ${ }^{12}$ und schnell bewegte Atomkerne mit Energien $>10^{9} \mathrm{eV}$ (kosmische Strahlung) ${ }^{13}$ ausgesandt werden, so ergibt sich aus der Annahme der Existenz intensiver Eruptionen auf den RW Aurigae-Sternen die Hypothese, daß ein großer Anteil der galaktischen Radiofrequenz-Strahlung und der kosmischen Strahlung auf solchen Sternen entsteht. Im Zusammenhang mit der Himpelschen Ansicht über die Ursache der Eiszeiten $\left(\mathrm{Himpel}^{6}\right)$ ergäbe sich, daß in den Eiszeiten die kosmische Strahlung wesentlich verstärkt gewesen sein muß. Daraus resultieren interessante Einblicke in die Phylogenie und namentlich in den Prozeß der Neuentstehung ganzer Tierklassen, über die der Verf. an anderer Stelle referiert hat ${ }^{14}$.

darin, daß die Nebelveränderlichen von außen und die $R$ Cor. bor.-Sterne von innen aufgeheizt werden.

12 A. C. B. Lovell u. C. J. Banwell, Nature [London] 158, 517 [1946].

${ }_{13}$ S. E. Forbush, Physic. Rev. 70, 771 [1946]; A. Ehmert, Z. Naturforschg. 3a, 264 [1948].

${ }^{14}$ H. -J. Binge, , Über die vermutliche Ursache der Korrelation zwischen der phylogenetischen Entwicklung der Säugetiere und Klimawechseln in geologischer Vergangenheit". Veröffentlichungen des Instituts für Physikal.-biolog. Lichtforschung, Hamburg 1949. In dieser Arbeit ist bereits eine kurze Zusammenfassung der Fleckenentstehungshypothese des Verf. enthalten. 